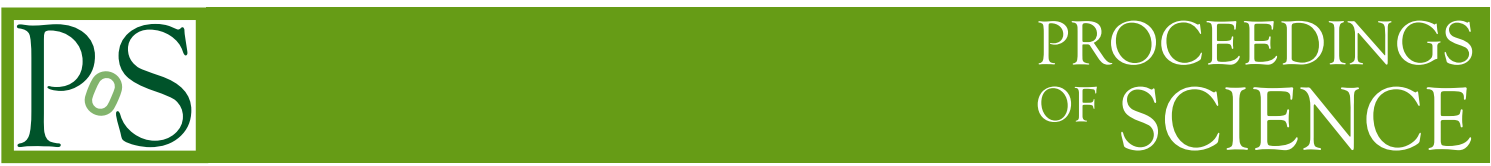

\title{
OH masers in the Milky Way and Local Group galaxies in the SKA era
}

\author{
Sandra Etoka*1, Dieter Engels ${ }^{1}$, Hiroshi Imai ${ }^{2}$, Joanne Dawson ${ }^{3}$, Simon Ellingsen ${ }^{4}$, \\ Lorant Sjouwerman ${ }^{5}$, Huib van Langevelde ${ }^{6,7}$ \\ ${ }^{1}$ Hamburger Sternwarte, ${ }^{2}$ Kagoshima University, ${ }^{3}$ Macquarie University, ${ }^{4}$ University of \\ Tasmania, ${ }^{5}$ National Radio Astronomy Observatory, ${ }^{6}$ Joint Institute for VLBI in Europe, \\ ${ }^{7}$ Sterrewacht Leiden \\ E-mail: sandra.etoka@googlemail.com
}

\begin{abstract}
The intense line emission of $\mathrm{OH}$ masers is a perfect tracer of regions where new stars are born as well as of evolved stars, shedding large amounts of processed matter into the interstellar medium. From SKA deep surveys at $18 \mathrm{~cm}$, where the maser lines from the ground-state of the $\mathrm{OH}$ molecule arise, we predict the discovery of more than 20000 sources of stellar and interstellar origin throughout the Galaxy. The study of this maser emission has many applications, including the determination of magnetic field strengths from polarisation measurements, studies of stellar kinematics using the precisely determined radial velocities, and distance determinations from VLBI astrometry. A new opportunity to study shocked gas in different galactic environments is expected to arise with the detection of lower luminosity masers. For the first time, larger numbers of $\mathrm{OH}$ masers will be detected in Local Group galaxies. New insights are expected in structure formation in galaxies by comparing maser populations in galaxies of different metallicity, as both their properties as well as their numbers depend on it. With the full capabilities of SKA, further maser transitions such as from excited $\mathrm{OH}$ and from methanol will be accessible, providing new tools to study the evolution of star-forming regions in particular.
\end{abstract}

Advancing Astrophysics with the Square Kilometre Array

June 8-13, 2014

Giardini Naxos, Italy

\footnotetext{
* Speaker.
} 


\section{Introduction}

In the Milky Way, maser emission is often observed in the circumstellar shells of red giant stars and in the surroundings of young stellar objects (YSOs). These are environments which are cool enough to form molecules and provide sufficient velocity coherence and density, so that the masers are naturally excited. The strongest stellar masers are those of oxygen-bearing molecules like hydroxyl $(\mathrm{OH})$, water $\left(\mathrm{H}_{2} \mathrm{O}\right)$ and silicon-monoxide $(\mathrm{SiO})$. In addition, methanol $\left(\mathrm{CH}_{3} \mathrm{OH}\right)$ and to lesser extent, ammonia $\left(\mathrm{NH}_{3}\right)$ and formaldehyde $\left(\mathrm{H}_{2} \mathrm{CO}\right)$ masers are detected close to starformation regions (SFRs). The major maser transitions accessible with the SKA during Phase 1 will be from $\mathrm{OH}$ at frequencies of 1612, 1665 and $1667 \mathrm{MHz}$ in band 2 and $1720 \mathrm{MHz}$ in band 3.
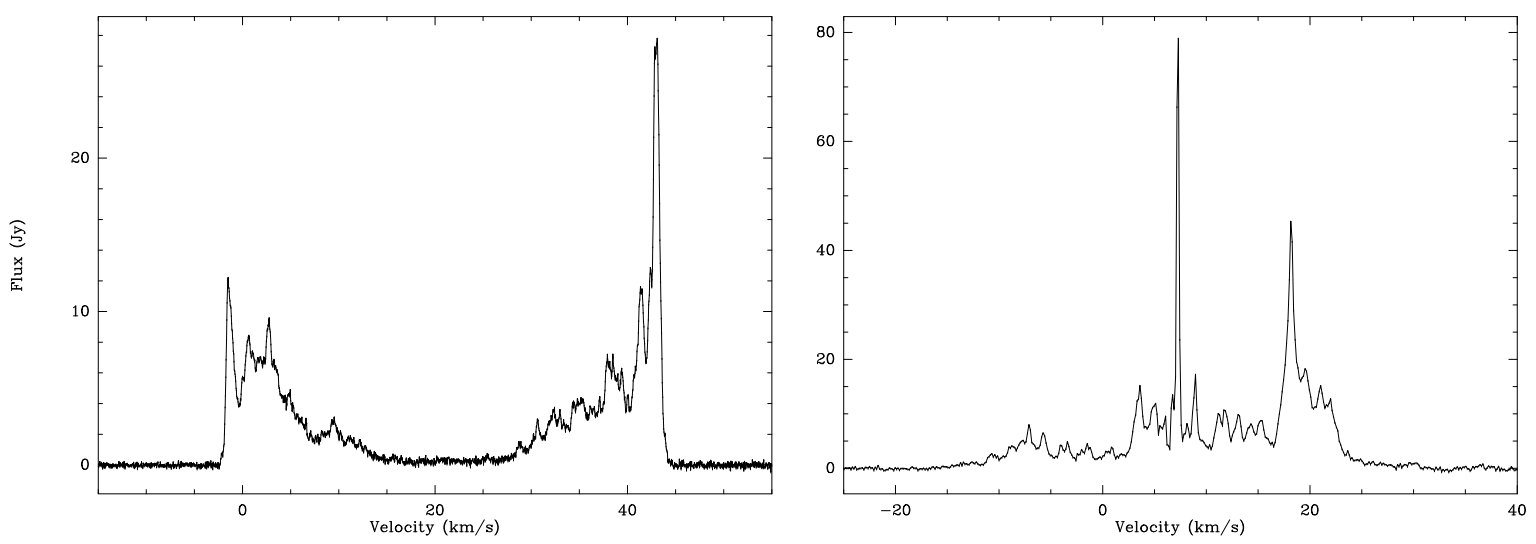

Figure 1: Left: 1612-MHz OH maser of the long-period variable star $\mathrm{OH}$ 16.1-0.2 Right: $1665-\mathrm{MHz} \mathrm{OH}$ maser of the star-forming region Orion KL. Both spectra were taken with the Nançay radiotelescope.

Known $\mathrm{OH}$ masers in the Galaxy are typically located in the plane at distances 2-8 kpc. The evolved-star and star-forming-region origin can be differentiated from their spectral shapes and the ratio of intensities between the transitions.

Evolved stars are generally strongest in the 1612-MHz transition and have a spectral shape dominated by two peaks with a separation of $30 \pm 10 \mathrm{~km} \mathrm{~s}^{-1}$ (e.g. Fig. 1 left panel). The shape is due to the origin of the masers in an expanding circumstellar shell where the strongest amplification occurs radially. The two strong peaks then come from the front and the back sides of the shell, and the radial velocity of the star and the expansion velocity of the shell can be determined directly from the central velocity of the double-peaked profile and the velocity interval covered by the emission. The evolved-star masers vary in phase with the stellar luminosity variations. Superposed on this are peculiar variations due to flares or intervals of generally low emission levels.

All 4 ground-state lines can be observed towards SFRs, though the main-lines (1665 and $1667 \mathrm{MHz}$ ) are the most commonly observed. Typically, SFR maser spectra are composed of narrow spectral components $\left(\leq 1-2 \mathrm{~km} \mathrm{~s}^{-1}\right)$ which can spread over a wide range of velocity depending on the complexity of the SFR. The intensity of the individual components can vary by up to four orders of magnitude within the same SFR complex (e.g. Fig. 1 right panel showing the rich 1665-MHz spectrum towards the Orion-KL SFR complex). Often, these spectra show substan- 
tial polarisation (including Zeeman pattern signatures) as well as substantial temporal variability (including flares) and periodic behaviours have also even been recorded towards class II 6.7-GHz methanol masers (e.g. Goedhart, Gaylard \& van der Walt 2004).

Masers originating from SFRs and supernova remnants (SNRs) are commonly referred to as "interstellar masers". This term shall be used hereafter, bearing in mind that we are focusing here on the evolved-star and SFR populations. The differentiation between SFRs and SNRs will be made to remove any ambiguity when appropriate.

\section{SKA survey framework}

\subsection{Luminosity distributions of galactic $\mathrm{OH}$ masers}

Currently >2000 stellar (Engels \& Bunzel, 2014; hereafter EB14) and several hundred interstellar OH masers are known in the Milky Way. Most were discovered by surveys with single-dish radiotelescopes with typical survey limits of several hundred mJy. The most comprehensive survey for stellar OH masers so far, is the ATCA/VLA OH 1612-MHz survey (Sevenster et al. 2001), covering the Galactic plane at $|l| \leq 45^{\circ}$ and $|b| \leq 3^{\circ}$. From this survey, we know that the number of detections is still increasing with increasing sensitivity.

A series of surveys for interstellar masers were made by Caswell, Haynes \& Goss (1980); Caswell \& Haynes (1983a\&b and 1987), Caswell (1998) and Caswell, Green \& Phillips (2013, 2014). They cover the Galactic plane to within 1 degree, in the range $233^{\circ} \leq l \leq 60^{\circ}$, and extend to masers slightly weaker than $1 \mathrm{Jy}$ for the 1980 s series of survey and $\sim 0.2 \mathrm{Jy}$ for the more recent series. The ongoing Southern Parkes Large-Area Survey (SPLASH) for OH masers, which has a mean 5-sigma flux limit of $0.3 \mathrm{Jy}$, has already doubled the number of known masers for the area in common with previous surveys (Dawson et al. 2014).

To estimate the yields of more sensitive surveys, we have constructed distributions of specific $\mathrm{OH}$ luminosities $L_{v}=f_{\text {peak }} \cdot 4 \pi D^{2}$, with $f_{\text {peak }}$ the peak flux density of the strongest feature in the maser spectrum and $D$ the kinematic distance. The distribution of flux densities and specific luminosities for stellar sources, taken from EB14, are shown in the first row of Fig. 2. For the interstellar masers we used the flux densities from the survey of Caswell and collaborators, and calculated specific luminosities using the kinematic distances provided by them. In case of ambiguity we used the distance quoted as 'more likely'. The distributions obtained for the interstellar sources are shown in the second row of Fig. 2.

The flux distributions are peaking at $\sim 0.5 \mathrm{Jy}(1612 \mathrm{MHz})$ for stellar and at $\sim 4 \mathrm{Jy}(1665 / 67 \mathrm{MHz})$ for interstellar masers, reflecting the different sensitivities of the contributing surveys. The luminosity distributions peak at $2 \times 10^{15} \mathrm{Watt}_{\mathrm{Hz}^{-1}}$ and $\sim 10^{16} \mathrm{Watt}_{\mathrm{Hz}}^{-1}$ for the two different maser origins. The decrease in number at lower luminosities is very likely due to incompleteness, and this will be probed by future surveys.

\subsection{Implications of maser variability on surveys}

Due to the variability of the masers, single epoch surveys detect only a fraction of the full population. Detection rates less than $50 \%$ are common for targeted surveys toward infrared selected samples of red giants. The missing detections cannot be explained by sensitivity limitations alone, 

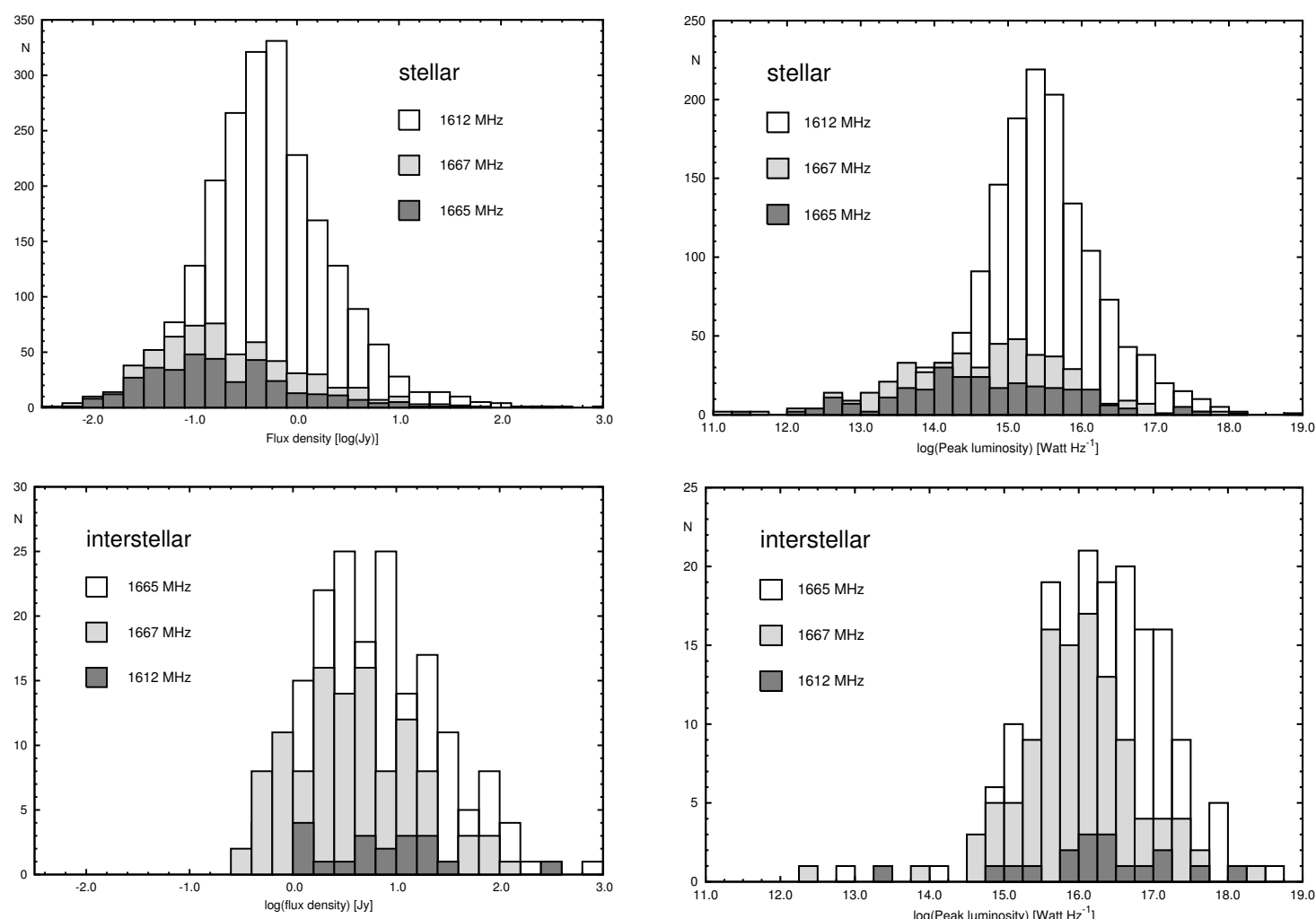

Figure 2: Left panel: Distribution of peak flux densities for stellar masers (top) and for interstellar masers (bottom). Increasing shading denotes the 1612, 1667, $1665 \mathrm{MHz}$ transition for stellar and 1665, 1667, $1612 \mathrm{MHz}$ transition for interstellar masers. Right panel: Distribution of corresponding peak luminosities assuming kinematic distances for stellar (top) and interstellar masers (bottom).

but most likely are due to variability on a range of timescales. On timescales of years, the stellar masers vary in response to the luminosity variations of the parent star. Repeated observations usually produce detections for such cases. Part of the O-rich population however are persistently not detected, although 'maser' and 'non-maser' AGB stars show no differences in their optical or infrared properties. The fraction $\eta$ of 'maser' AGB stars is dependent on infrared colour (e.g. mass-loss rates) and ranges between 10 and $60 \%$ for a flux density limit of $\approx 50 \mathrm{mJy}$ (Lewis, 1992).

Either these 'non-maser' stars possess only low-luminosity masers or the distribution of maser sites in their circumstellar shells is not spherically symmetric, so that only those stars are detected in which the $\mathrm{OH}$ maser emission is beamed toward Earth. Such issues are also valid for interstellar masers. For stellar masers, the beaming directions may change on timescales related to the crossing time through the shell ( $\sim 1000$ years). Therefore, we predict that the coming $\mathrm{OH}$ maser surveys, which will be separated $\sim 30$ years from the historical ones, are also expected to discover new bright $(\gg 1 \mathrm{Jy}$ ) masers. The rate of new discoveries in stars not detected previously are a direct test of the timescales on which reconfigurations in the expanding circumstellar shells may occur.

\subsection{The Galactic OH maser population}

A rough estimate of the yield of future surveys for stellar $\mathrm{OH}$ masers can be made for $1612 \mathrm{MHz}$ 
using the model of Jackson et al. (2002) for the Galactic distribution of AGB stars. This model is based on the analysis of the Galactic distribution of $\approx 10000$ IRAS sources with colours appropriate for mass-losing AGB stars. It predicts $\approx 200000$ AGB stars in the Milky Way. 1612-MHz OH flux densities can be estimated on the basis of dust mass-loss rates $\dot{M}_{d u s t}\left(\right.$ in $M_{\odot} \mathrm{yr}^{-1}$ ) from the relation $f_{O H}=3.15 \times 10^{9} \dot{M}_{\text {dust }}\left(v_{\text {exp }} D^{2}\right)^{-1}$ (Zijlstra et al. 1996, taking into account the factor 2 needed for a better fit of the LMC data as predicated by Marshall et al. 2004), where $f_{O H}$ is the peak flux density in Jy, $v_{\text {exp }}$ the expansion velocity in $\mathrm{km} / \mathrm{s}$, and $D$ the distance in $\mathrm{kpc}$. For a given volume in the Galaxy, the space density of AGB stars was obtained from the Jackson et al. model, and it was assumed that the stars have a fixed IRAS colour distribution. The IRAS colours were converted to $\dot{M}_{\text {dust }}$ using the pure silicate models of Groenewegen (2006), which predict infrared colours for a range of gas mass-loss rates for fixed bolometric luminosity $L=3000 L_{\odot}, v_{\exp }=10 \mathrm{~km} \mathrm{~s}^{-1}$, and a gas-to-dust ratio $\Psi=200$. From the dust mass-loss rates, $\mathrm{OH}$ flux densities were obtained as described above.
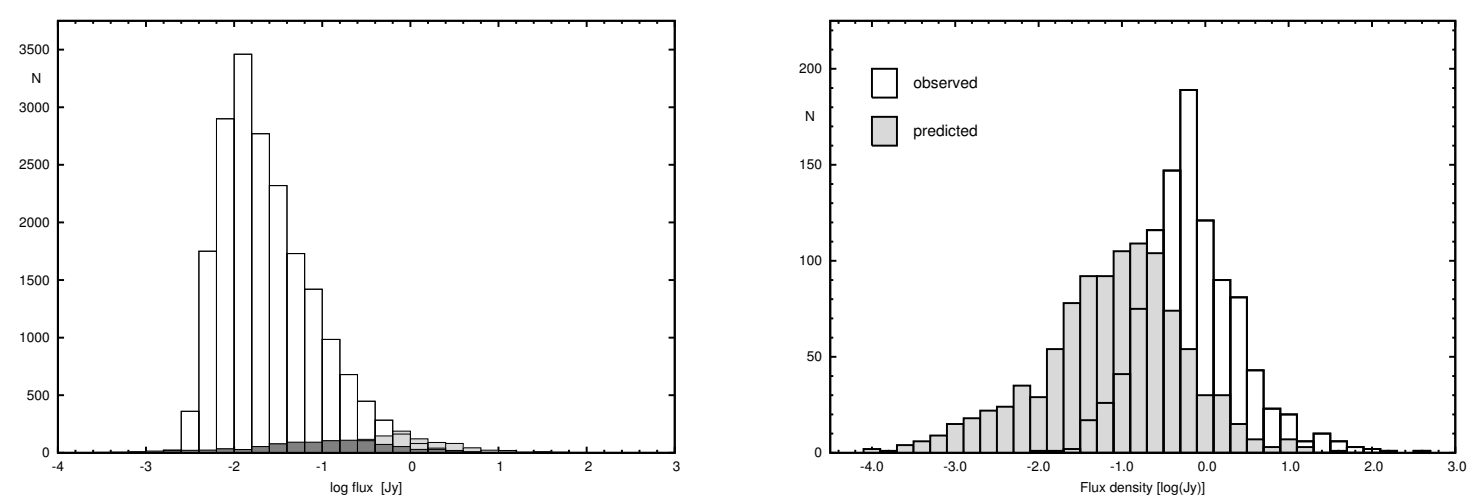

Figure 3: Left panel: Predicted distribution of 1612-MHz stellar $\mathrm{OH}$ maser flux densities based on the model of the Galactic distribution of AGB stars of Jackson et al. (2002) and a relation between dust massloss rates and $\mathrm{OH}$ maser luminosities from Marshall et al. (2004). The shaded distributions are from the right panel. Right panel: Observed stellar $\mathrm{OH}$ maser population in the front part of the solar Galactic hemisphere (that is up to a distance of $D \cdot \cos (l) \leq 8.0 \mathrm{kpc}$ ) and its predicted counterpart in the anti-solar Galactic hemisphere (see Sect. 3.2). The observed population is taken from the EB14 catalogue.

The actual numbers of $\mathrm{OH}$ masers predicted depend critically on the distribution of the massloss rates (or IR colours) among the AGB star population and also on the fraction $\eta$ of AGB stars actually showing a maser with the predicted luminosity. The observed IRAS colour distributions are biased towards higher mass-loss rates, because these stars emit most of their energy in the midinfrared. Corrections have to be estimated therefore for low $\dot{M}_{d u s t}$. The range of possible $\dot{M}_{d u s t}$ distributions and the fraction $\eta$ of $\mathrm{OH}$ emitting AGB stars was constrained by the requirement that the model reproduces the results of the survey of Sevenster et al. (2001), e.g. the number of detections down to the survey limit of $\approx 150 \mathrm{mJy}$ and the flux distribution.

The result for $\eta=0.1$ is shown in Fig. 3 (left panel), giving $\approx 11000$ stellar $\mathrm{OH} 1612-\mathrm{MHz}$ maser with $F_{O H} \geq 10 \mathrm{mJy}$ in the Galactic plane $(|b|<3 \mathrm{deg})$. This value of $\eta$ is at the lower end of the range observed by Lewis (1992), but includes also by default 'non-maser' carbon-rich AGB stars in the population. Higher estimates can be created by adopting a steeper colour distribution, demanding $\eta>0.1$ to reproduce the observed high-luminosity masers. Accordingly, the prediction 
of the number of fainter masers will be scaled up.

In addition to the predicted number of stellar masers, an unknown number of interstellar masers will be present in the Galactic plane. Some of these masers will be detected prior to the SKA by surveys such as GASKAP. This survey aims to reach an rms for point sources of $1 \mathrm{mJy}$ in the Galactic plane and of $0.5 \mathrm{mJy}$ in the Magellanic Clouds. However, the angular resolution will only be $\sim 20$ arcsec (Dickey et al. 2013), and thus the masers at the lowest flux limits may not be distinguishable from thermal extended emission. From a simple extrapolation of the number of identified maser sources in the pilot SPLASH region $\left(\sim 200\right.$ sources within an area $10^{\circ}$ width in Galactic Longitude), it is expected that at the SPLASH 5-sigma flux limit of $0.3 \mathrm{Jy}$ several thousand maser sources are detectable. The number of interstellar masers detectable down to $F_{O H} \geq 10 \mathrm{mJy}$ is therefore at least as large as that predicted for the stellar masers, yielding a total number of detectable masers in the Galactic plane of $\geq 20000$.

\section{The impact of $\mathrm{OH}$ maser surveys with SKA1}

Adopting the specific luminosity distributions from Fig. 2 as representative for the stellar and interstellar maser luminosity distributions in the Galaxy, sensitivity limits for an SKA survey to detect a major part of these maser populations at different distances can be estimated. Adopting

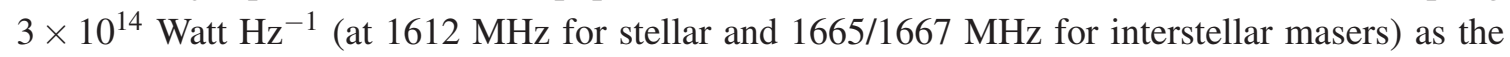
lower limit for the maser population to study, and a detection threshold of $\mathrm{S} / \mathrm{N}=10$, sensitivity limits $F_{\text {lim }}=4,1$, and $0.1 \mathrm{mJy}$ are required to probe the population at distances of 8,16 and $50 \mathrm{kpc}$ respectively. These correspond to the Galactic center, the anti-solar, and the Large Magellanic Cloud distances.

Most of the ground-state $\mathrm{OH}$ maser surveys undertaken in the Milky Way have been made with an angular resolution of typically $20 \mathrm{arcsec}$ and GASKAP will deliver a similar resolution. A new SKA1 survey, with a resolving power 20 times that of GASKAP, will allow a direct comparison of the other side of the Galaxy, LMC \& SMC stellar and interstellar maser populations at resolution roughly similar to Galactic surveys.

The 3 ground-states lines at 1612, 1665 and $1667 \mathrm{MHz}$ are simultanously covered by Band 2 with SKA1-MID and SKA1-SUR and the $4^{\text {th }} \mathrm{OH}$ ground-state line at $1720 \mathrm{MHz}$ can be accessed with Band 3. While SKA1-SUR will offer an unprecedented survey speed, SKA1-MID, with a SEFD 4 times better than SKA1-SUR as well as an increase by a factor of 4 in the power of resolution ( 0.22 compared to 0.9 arcsec respectively), will be better suited for investigating the structure of the maser emission to the same resolution as e.g. eMERLIN. SKA1-MID Band 5 would offer the ability of accessing the 6-GHz excited-OH maser transitions, as well as the class-II 6.7-GHz methanol maser transition associated exclusively with the formation process of high-mass stars.

In the following sections, OH maser surveys rendered possible with SKA1-MID and SKA1SUR capabilities are discussed. They are summarised in Table 1. 
Table 1: SKA1 maser surveys

\begin{tabular}{|c|c|c|c|c|c|c|}
\hline $\begin{array}{l}\text { SKA1 Survey } \\
\text { Name }\end{array}$ & $\begin{array}{l}\text { Sensitivity } \\
(\mathrm{mJy})\end{array}$ & $\begin{array}{l}\text { Int. per }{ }^{1} \\
\text { pointing } \\
\text { (hours) }\end{array}$ & Coverage & $\begin{array}{l}\text { Pointing } \\
\mathrm{Nb}\end{array}$ & $\begin{array}{l}\text { Total }^{1,2} \\
\text { time } \\
\text { (hours) }\end{array}$ & Aim \\
\hline SUR-All-Sky & 4 & 0.11 & $21000 \mathrm{deg}^{2}$ & 1300 & 143 & All-Sky shallow survey \\
\hline SUR-Shallow & 1.0 & 1.75 & $\begin{array}{l}-60^{\circ} \leq l \leq+60^{\circ} \\
b \leq 4^{\circ}\end{array}$ & 30 & 52.5 & $\begin{array}{l}\text { Anti-solar Galactic } \\
\text { hemisphere maser pop. }\end{array}$ \\
\hline SUR-Deep & 0.3 & 19 & $\begin{array}{l}-60^{\circ} \leq l \leq+60^{\circ} \\
b \leq 4^{\circ}\end{array}$ & 30 & 570 & $\begin{array}{l}\text { Seach for low } \\
\text { luminosity pop. }\end{array}$ \\
\hline SUR-LMC & 0.1 & 175 & $11 \times 10 \operatorname{deg}^{2,3}$ & 9 & 1575 & LMC survey (3-sigma) \\
\hline SUR-SMC & 0.1 & 175 & $5.5 \times 3.5 \mathrm{deg}^{2,3}$ & 4 & 700 & SMC survey (3-sigma) \\
\hline MID-MC & 0.05 & 40 & & 100 & 4000 & $\begin{array}{l}\text { LMC+SMC confirmation } \\
\text { targeted survey (6-sigma) }\end{array}$ \\
\hline MID-GAL & 0.05 & 40 & $1 \mathrm{deg}^{2}$ & 4 & 160 & Triangulum Survey \\
\hline
\end{tabular}

1: for a channel width of $5 \mathrm{kHz} \Leftrightarrow \sim 0.9 \mathrm{~km} / \mathrm{s}$; SEFD MID $_{1}=1.7$ (cf. Table 1 of SKA-TEL-SKO-DD-001 (Dewdney et al. 2013); corresponding to "combined": 190 antennas + Meerkat); $\operatorname{SEFD}_{\mathrm{SUR}}=7.1$

2: $\mathrm{FOV}_{\mathrm{MID}}=0.5 \mathrm{deg}^{2} ; \mathrm{FOV}_{\mathrm{SUR}}=18 \mathrm{deg}^{2}$

3: from the NED (http://ned.ipac.caltech.edu/)

\subsection{OH masers in the anti-solar Galactic hemisphere}

To estimate the likely range of flux densities of stellar $\mathrm{OH}$ masers in the Milky Way located beyond the Galactic center (hereafter the anti-solar Galactic hemisphere), we used all stellar masers with kinematic distance estimates from EB14 with $D \cdot \cos (l) \leq 8.0 \mathrm{kpc}$, e.g., the population of stellar masers in the solar Galactic hemisphere. We assumed that the population in the anti-solar Galactic hemisphere is a mirror of the front part population and calculated their flux densities as if observed from the Sun. The result is shown in Fig. 3 (right panel), where the open bins represent the observed population and the shaded bins represent the predicted population in the anti-solar Galactic hemisphere. The bulk of the predicted population has flux densities between 10 and $500 \mathrm{mJy}$ in good agreement with the maximum of the flux density distribution obtained from the prediction presented in Sect. 2.3. This population can be detected in $\sim 50$ hours with SKA1-SUR (cf. SUR-Shallow in Table 1).

\subsection{Low-luminosity masers}

Surveys of increasing sensitivity in the Galaxy will not only extend the distances out to which masers will be detected, but will also extend the maser luminosity range towards lower levels than before. It is unknown whether the specific luminosities of masers have lower limits. A plausible assumption is that there is no such limit. A good illustration of this issue is the $\mathrm{OH}$ flaring Mira population (Etoka \& Le Squeren 1997). $o$ Ceti is an ideal example amongst these stars, as it shows absence of detectable $\mathrm{OH}$ maser emission for decades followed by long-lasting $\mathrm{OH}$ flaring events (Etoka et al. 2010a). The mean intensity of the OH flaring emission towards $o$ Ceti is $\sim 2 \mathrm{Jy}$, corresponding to a luminosity of $\sim 2 \times 10^{12} \mathrm{Watt}_{\mathrm{Hz}^{-1}}$, which can be taken as a typical threshold for this type of stellar maser population. The dichotomy 'maser' and 'non-maser' O-rich AGB stars would then vanish with increasingly sensitive surveys. In addition, it seems also plausible that $\mathrm{OH}$ maser emission will be discovered in classes of objects, which do not, or rarely possess 
high-luminosity masers. One example are Planetary Nebulae (PNe). There are only six sources in which the presence of (1612 MHz) OH maser emission has been confirmed (Uscanga et al. 2012). Another example is the so far unique detection of 1720-MHz maser emission from an evolved star (made towards the post-AGB star OH 9.097-0.392 by Sevenster \& Chapman 2001). In post-AGB stars and $\mathrm{PNe}$, the maser emission is probably related to shock-excitation in the interface between a fast post-AGB wind with a remnant slow AGB wind (Etoka et al. 2009). These excitation events might be short-lived, might lead to beamed emission or produce only low-luminosity maser emission. It seems likely, that new sensitive surveys will discover many new masers in stars already evolved away from the AGB, allowing us to study the bipolar outflows emerging in this phase in more detail. Towards SFRs, faint ground-state $1665 / 1667 \mathrm{MHz}$ OH maser from young stars which will not ionize their environment has been found (e.g. the Turner-Welch object detected in the W3(OH) SFR complex by Argon, Reid \& Menten 2003). A 140-hours survey over the complete sky accessible by SKA1-SUR (cf. SUR-All-Sky in Table 1) with a flux limit of 4 mJy will probe the presence of a population of low-luminosity masers (e.g. $\mathrm{L}_{\mathrm{OH}}<3 \times 10^{14} \mathrm{~W} \mathrm{~Hz}^{-1}$ ) among evolved stars and SFRs in the solar vicinity, which previous surveys have only investigated for higher luminosity masers. A deeper blind search for such low-luminosity masers in the inner Galactic disk could be achieved within $\sim 570$ hours (cf. SUR-Deep in Table 1).

\subsection{Galactic analog $\mathrm{OH}$ masers in galaxies of the Local Group}

Late-type star evolution and the star formation process may differ in galaxies with other metallicity environments. The extension of the Galactic maser research to galaxies in the Local Group will provide another tool to study these differences. Due to their proximity, the Magellanic Clouds with their low-metallicity environment are the first galaxies to address.

Only a small number of maser sources with luminosities similar to their Galactic analogs are known outside the Milky Way. In the LMC, ten 1612-MHz OH masers with peak-flux densities $17-$ $600 \mathrm{mJy}$ are known in evolved stars (Marshall et al. 2004). These low-metallicity, high-mass LMC stars have contributed significantly to our understanding of the expansion velocities as indicators of metallicity. A few searches for ground-state $\mathrm{OH}$ maser of interstellar origin have been made in the 1980's and 1990's. A handful of 1665-MHz maser sources (sometimes accompanied with fainter $1667 \mathrm{MHz}$ ) have been detected towards the LMC (Brooks \& Whiteoak 1997). A similar number of detections of 1720-MHz maser emission originating from SNRs have also been found towards the LMC (Brogan et al. 2004). Regarding the SMC, to date, OH masers have not been detected. Furthermore, maser emission of evolved-star origin has not been detected so far, and the number of maser sources of SFR origin detected is very low (in total $6 \mathrm{H}_{2} \mathrm{O}$ masers of SFR origin: Scalise \& Braz 1982; Breen et al. 2013; also, the systematic survey done by Green et al. (2008) failed to detect any class II 6.7-GHz methanol masers).

With the sensitivities provided by SKA1, the options for maser research in the Magellanic Clouds may dramatically increase. To verify our ideas about Galactic structure evolution, it would be of great importance to compare the populations of stellar masers in the Galaxy with those in other galactic environments. Currently known stellar masers in the LMC were discovered by targeted searches towards luminous and mid-infrared bright sources. Their specific luminosities $L_{O H}$ cover the range $15.50<\log L_{O H}<17.50$, which corresponds to the bright tail of the luminosity 


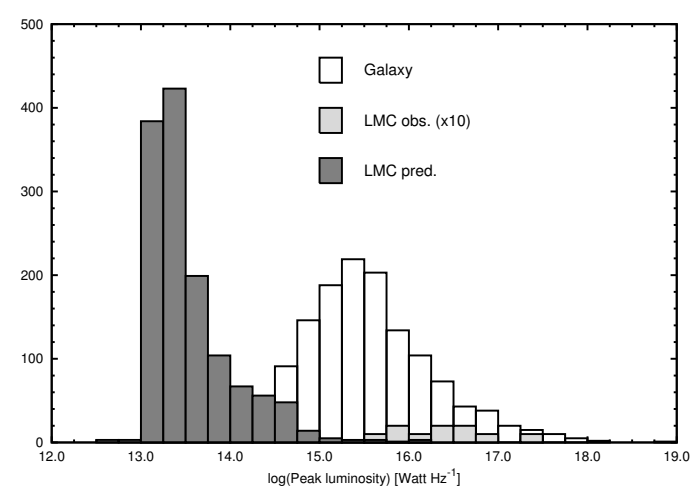

Figure 4: Predicted specific luminosity distribution for 1612-MHz OH masers in the LMC. The prediction is compared to the luminosities of the observed masers in the LMC (numbers are multiplied by 10) and the distribution of known masers in the Galaxy from the EB14 catalogue.

distribution in the Galaxy (cf. Fig. 4). An inventory of evolved stars in the LMC $\left(\sim 50 \mathrm{deg}^{2}\right)$ and the SMC $\left(\sim 30 \mathrm{deg}^{2}\right)$ has been provided by the SAGE-Spitzer survey (Meixner et al. 2006, Gordon et al. 2011) in the infrared. In the LMC, depending on the photometric classification according to chemistry, 20000-25000 of them are expected to be oxygen-rich (Riebel et al. 2012). For most stars, the dust mass-loss rates given by Riebel et al. are low $\left(\dot{M}_{\text {dust }}<5 \times 10^{-10} M_{\odot} \mathrm{yr}^{-1}\right)$ and their circumstellar shells do not qualify as $\mathrm{OH}$ maser hosts. To estimate the $\mathrm{OH}$ maser luminosities of the $\sim 1300$ stars with higher dust mass-loss rates we used the relation from Sect. 2.3 relating $\dot{M}_{\text {dust }}$ with 1612-MHz OH maser flux densities $f_{O H}$. Assuming $v_{\text {exp }}=14 \mathrm{~km} \mathrm{~s}^{-1}$ and $\mathrm{D}=50 \mathrm{kpc}$, the luminosity distribution shown in Fig. 4 is obtained. The result indicates that most bright masers in the LMC are already known, and the luminosities of the masers to be discovered will be lower than that of the bulk of the known Galactic masers. The reason for this is the classification of almost all stars with high mass-loss rates ("extreme AGB stars") as C-stars by the SAGE-Spitzer survey. The validity of this photometric classification will be directly probed by an $\mathrm{OH}$ maser survey of the LMC covering the full AGB population.

As expected, the number of masers in Fig. 4 increases for lower luminosities, following the increase of the number of AGB stars with decreasing mass-loss rates. With a survey limit of $0.1 \mathrm{mJy}$

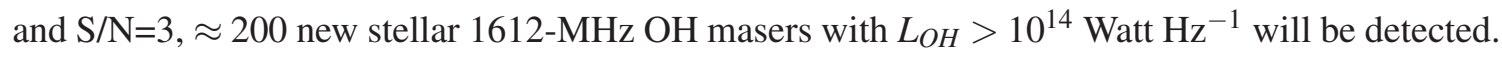
These numbers should be seen as upper limits, if detection rates as in the Galaxy apply. On the other hand, more 1612-MHz OH masers might be found if there are more O-rich AGB stars among the "extreme AGB stars" than expected. Furthermore, since (preferentially main-line) masers are commonly present in the vicinity of Galactic YSOs, they are probably present around their LMC analogs. A mid-IR photometry search for high- and intermediate-mass YSOs in the LMC has been made by Gruendl \& Chu (2009) yielding $>1000$ sources. The number of OH masers detectable in the LMC with a $0.1 \mathrm{mJy}$ survey limit is therefore well above 200.

The number of O-rich evolved stars qualifying as potential $\mathrm{OH}$ maser hosts in the SMC is $>2000$ (Boyer et al., 2011), about 10\% of the LMC. Assuming, that the number of YSOs is also $10 \%$ of the corresponding number in the LMC, and allowing for the larger distance ( $\mathrm{D}=60 \mathrm{kpc})$, about two dozen new $\mathrm{OH}$ masers might be found, adopting the same survey limit as in the LMC. They will be the first ever detected in this galaxy. Both Magellanic Clouds can be surveyed in $\sim 2300$ hours for the $0.1 \mathrm{mJy}$ flux limit required (SUR-LMC, SUR-SMC in Table 1). Follow-up observations to improve the spatial resolution and the signal-to-noise ratio of the most interesting new discoveries are foreseen using SKA1-MID, adopting 100 pointings as a figure of merit (MID- 


\section{MC in Table 1).}

The recent interest in the evolved stellar population and in the mass-loss and age-metallicity relation in the Sagittarius Dwarf Spheroidal (Lagadec et al. 2008 \& 2009), located at only $25 \mathrm{kpc}$ midway between the Galaxy and the LMC, has prompted searches for $\mathrm{OH}$ masers in this dwarf galaxy. However, the current sensitivity limits are barely sufficient to perform statistical comparisons with evolved stars in the Galaxy and other nearby different metallicity systems. Such studies clearly have to wait for SKA.

Triangulum (M33) is the third largest member of the local group, and with $\delta=30.6^{\circ}$ is accessible with the SKA. Three $\mathrm{H}_{2} \mathrm{O}$ masers are known in this galaxy (Brunthaler et al. 2006), while searches for methanol masers (Goldsmith et al. 2008) and OH masers (Fix \& Mutel 1985; Baan, Haschick \& Henkel 1992) have to date been unsuccessful. The limiting sensitivity of the OH observations was $\approx 15 \mathrm{mJy}(5 \sigma)$, which sets an upper limit of $1.5 \times 10^{18} \mathrm{Watt}_{\mathrm{Hz}}^{-1}$ on the luminosity of potential M33 OH masers, adopting a distance of $\approx 880 \mathrm{kpc}$. The non-detection of $\mathrm{OH}$ masers in previous observations is not surprising, as only a small number of Galactic $\mathrm{OH}$ masers have luminosities in excess of this limit. We expect however that the brightest interstellar and the bright stellar $\mathrm{OH}$ masers, with $L_{\mathrm{OH}}>5 \times 10^{15} \mathrm{Watt} \mathrm{Hz}^{-1}$, among the more than 10000 AGB stars identified by Cioni et al. (2008) in M33 will be detectable with a survey (MID-GAL in Table 1), having the same sensitivity to that proposed for the LMC+SMC follow-up observations.

The study of $\mathrm{OH}$ masers with SKA in galaxies beyond the Local Group will focus on $\mathrm{OH}$ megamaser emission. This emission is $10^{8}$ times more luminous than Galactic analog $\mathrm{OH}$ masers, and is observed towards luminous and ultra-luminous infrared galaxies. These megamaser-hosting galaxies are either mergers or show evidence of interaction with other galaxies and are associated with a burst of star formation. Hence, megamasers are useful probes of the conditions where star formation is taking place in such galaxies as well as allowing extragalactic magnetic field measurements (McBride, Heiles \& Elitzur 2013, Robishaw, Quartaert \& Heiles 2008, cf. also Beswick et al. 2015 \& Robishaw et al. 2015).

\section{Survey applications}

\subsection{Polarisation \& magnetic fields}

$\mathrm{OH}$ masers, characterised by their spectral narrowness and their high sensitivity to magnetic fields, can be strongly polarised and commonly exhibit Zeeman splitting. Hence, they are a particularly useful tool for polarisation properties studies and retrieving the magnetic field structure and strength of the medium they are probing. They can be used for this purpose towards both evolved-stars (Amiri, Vlemmings \& van Langevelde 2011; Etoka \& Diamond 2010b) and SFRs (Caswell, Hutawarakorn \& Reynolds 2011a,b; Asanok et al. 2010; Wright, Gray \& Diamond 2004a,b; Hutawarakorn et al. 2002). Not only can the Zeeman splitting be used to explore the magnetic fields from individual objects, but also as a whole throughout the Milky Way and the galaxies of the Local Group (Green et al. 2012, cf. also Robishaw et al. 2015).

\subsection{Stellar kinematics}

Similar to $\mathrm{SiO}$ masers, a large variety of stellar populations host $\mathrm{OH}$ masers: evolved stars in 
the Galactic thick disk, bulge, and globular clusters in the halo as well as young stellar clusters in the Galactic thin disk (Deguchi et al. 2004). A large sample of OH masers will reveal the velocity fields of these Galactic components. The high luminosity of the masers together with the radial velocity information provided, allow the study of the stellar kinematics in the various Galactic components (yielding for example evidence for a bar in the Milky Way: Habing et al. 2006). The information on the kinematics, supplemented with the age and metallicity of the stars, will point to common origins and clues for Galactic evolution models. These studies will be greatly enhanced by increasing numbers of southern sources.

\subsection{VLBI \& astrometry}

Among evolved stars, OH/IR stars are sizeable objects of typically 10000 AU when mapped in the ground-state OH (Etoka \& Diamond 2004, 2010b), and so are SFR complexes (Cohen et al. 2006). This typical size corresponds to $1.25 \operatorname{arcsec}$ at $8 \mathrm{kpc}$ and $0.2 \operatorname{arcsec}$ at $50 \mathrm{kpc}$. With an achievable resolution of 0.22 arcsec, SKA-MID will be able to map a good fraction of the OH/IR stars and SFR complexes detected at Galactic-center distances and beyond, as well as the strongest and most extended objects in the LMC. To derive distances, VLBI-Astrometry (Vlemmings \& van Langevelde 2007) or the "phase-lag technique" can be used (Engels et al. 2014). Since OH/IR stars can be found in all the Galactic components (cf. the previous subsection 4.2), as opposed to SFRs, and provided that the $\mathrm{OH}$ circumstellar shell is extended, the distances of these various components (and hence a better presentation of the galactic structure) can potentially be inferred using this method.

Admittedly, extended OH/IR stars can be significantly resolved out with baselines longer than $1000 \mathrm{~km}$ (Imai et al. 2013). Since the resolving power increases with frequency, higher frequency masers (i.e. in Band 5) in VLBI in-beam mode will allow high resolution astrometry and hence distance determinations with the possibility of a 3D mapping of the structure of our Galaxy but also that of the LMC and SMC (cf. Green et al. 2015).

\subsection{SNR \& the Galactic center circumnuclear Disk}

Whereas the 1612-MHz OH masers are predominantly seen in circumstellar environments and the mainline 1665- and 1667-MHz OH masers are signposts for SFRs, the collisionally pumped $1720-\mathrm{MHz} \mathrm{OH}$ maser is typically seen as the only $\mathrm{OH}$ transition outlining shocked environments. Small shocks, like in the formation of massive stars and in post-AGB outflows are currently difficult to detect and thus limit our understanding of these stellar evolutionary phases. However, the more energetic shocks generated by SNRs plowing into dense (molecular) clouds are readily recognised by bright 1720-MHz maser emission observed at the interaction regions (Claussen et al. 1997, Frail et al. 1996). With more sensitive observations available, less bright masers appear and make it possible to probe shocks in different evolutionary stages of stars. The construction of 3-dimensional velocity models of expanding SNRs and of dynamical structures like the circumnuclear disk in the Galactic center will be possible (Sjouwerman \& Pihlstrom 2008).

\subsection{Synergy between $\mathrm{HI}$ and $\mathrm{OH}$ surveys}

Recent studies have revealed weak extended HI shells around evolved stars (Libert, Gérard, \& Le Bertre 2007). While GASKAP will be able to provide a catalogue of such shells, the SKA 
higher angular resolution and sensitivity is needed for the study of these HI shells in greater detail. This will provide extra information on the mass-loss history and on the way stellar matter, enriched in elements produced in the stellar interior, is re-injected into the interstellar medium.

Since SFRs are converging and dispersing points of matter in the interstellar space, combining the $\mathrm{OH}$ maser distribution and the map of $\mathrm{HI}$ emission will provide a panoramic view of the lifecycle of matter in the Galaxy. GASKAP will pioneer this aspect through simultaneous surveys of $\mathrm{OH}$ and $\mathrm{HI}$ emission. SKA1 will allow a better correlation between $\mathrm{OH}$ masers and HI clumps by resolving the smaller structures of $\mathrm{HI}$ directly associated with the $\mathrm{OH}$ maser sources.

\section{Prospects of research with the full SKA enhanced capabilities}

Because of the complex non-linear nature of maser pumping, there is no simple means of inferring the physical conditions from observations from the maser intensity itself. Nonetheless, maser transitions are inverted for a range of physical conditions. Thus, combining various maser species and within the same species different transitions to study SFR regions is quite powerful as a probe of e.g. local density and temperature variations down to a few hundreds of AU (Etoka, Cohen \& Gray 2005). Hence the combined information can give us access to the different physical components structure (i.e., outflows or disks) around a given YSO (Etoka, Gray \& Fuller 2012).

As mentioned in section 3, Band 2, $3 \& 5$ will allow access to a wide range of maser transitions. Since different maser transitions have different sensivities, or dependencies on the physical conditions, the presence or absence of the various transitions is expected to change as the star formation region evolves. These relationships can only be determined through statistical population studies of the different maser transitions (e.g. Breen et al. 2010). The ability of the SKA and its pathfinder instruments to make rapid, sensitive surveys of the Galactic Plane will open up new opportunities to undertake such studies on scales which have not previously been possible.

$\mathrm{OH}$ masers are already known to be present towards a handful of PPNe-PNe in the Galaxy, but it could be that faint $\mathrm{OH}$ masers (e.g., from AGB OH-shell remnants) towards these objects have not been detected in previous surveys due to sensitivity. Similarly, excited $\mathrm{OH}$ maser has also been found towards a handful of evolved objects (Desmurs et al. 2010).

The sensitivity and frequency range covered by the full SKA, will allow us to increase the statistics on these "rare events" through a systematic survey, and identify when in the evolutionary stage this occurs and probe for other possible "new classes of masers".

\section{Acknowledgments}

This Chapter is dedicated to the late Jim Caswell, whose review helped to improve the text considerably. His extensive and systematic research on masers in star-forming regions was invaluable to evaluate the prospects of SKA for maser research.

\section{REFERENCES}

Amiri N., Vlemmings W. \& van Langevelde H.J., 2011, A\&A, 532, 149

Argon A., Reid M.J. \& Menten K.M., 2003, ApJ, 593, 925 
Asanok K., Etoka S., Gray M.D. et al., 2010, MNRAS, 404, 120

Baan W.A., Haschick A. \& Henkel C., 1992, AJ, 103,728

Beswick R., et al., 2015, "SKA studies of nearby galaxies: star-formation \& accretion processes across all environments", in proceedings of "Advancing Astrophysics with the Square Kilometre Array", PoS(AASKA14)070

Boyer M., et al., 2011, AJ 142, 103

Breen S.L., Ellingsen S.P., Caswell J.L. \& Lewis, B.E., 2010, MNRAS, 401, 2219

Breen S.L., Lovell J.E.J., Ellingsen S.P. et al., 2013, MNRAS, 432, 1382

Brogan C.L., Goss W.M., Lazendic J.S. \& Green A.J., 2004, ApJ, 128, 700

Brooks K.J. \& Whiteoak J.B., 1997, MNRAS, 291, 395

Brunthaler A., Henkel C., de Blok W.J.G. et al., 2006, A\&A, 457, 109

Caswell J.L., Haynes R.F. \& Goss M.W., 1980, Aust. J. Phys., 33, 639

Caswell J.L. \& Haynes R.F. 1983a, Aust. J. Phys., 36, 361

Caswell J.L. \& Haynes R.F. 1983b, Aust. J. Phys., 36, 417

Caswell J.L. \& Haynes R.F. 1987, Aust. J. Phys., 40, 215

Caswell J.L., 1998, MNRAS, 439, 1680

Caswell J.L., Kramer B.-Hutawarakorn \& Reynolds J.E., 2011a, MNRAS, 414, 1914

Caswell J.L., Kramer B.-Hutawarakorn \& Reynolds J.E., 2011b, MNRAS, 415, 3872

Caswell J.L., Green J.A. \& Phillips C.J., 2013, MNRAS, 431, 1180

Caswell J.L., Green J.A. \& Phillips C.J., 2014, MNRAS, 439, 1680

Cioni M.-R.L., Irwin M., Ferguson A.M.N. et al. 2008, A\&A, 487, 131

Cohen R.J., Gasiprong N., Meaburn J. \& Graham M.F., 2006, MNRAS, 367, 541

Claussen M., et al. 1997, ApJ, 489, 143

Dawson J., et al., 2014, MNRAS, 439, 1596

Deguchi S., Fujii T., Glass, I. et al. 2004, PASJ, 56, 765

Desmurs J.-F., Baudry A., Sivagnanam P., Henkel C., Richards A.M.S.\& Bains I., 2010, A\&A, 520, A45

Dewdney P., Turner W., Millenaar R., McCool R., Lazio J., Cornwell T., 2013, "SKA1 System Baseline Design", Document number SKA-TEL-SKO-DD-001, Revision 1

Dickey, J., et al. 2013, PASA, 30, 3

Engels D. \& Bunzel F., 2014, A\&A, submitted; Database of Circumstellar Masers v2.4, http://www.hs.uni-hamburg.de/maserdb

Engels D., Etoka S., Gérard E. \& Richards A.M.S., 2014, Proc. of the conference "Why galaxies care about AGB stars III", Eds. F.Kerschbaum, J. Hron \& B. Wing (in press)

Etoka S. \& Le Squeren A.M., 1997, A\&A, 321, 877

Etoka S. \& Diamond P.J., 2004, MNRAS, 348, 34

Etoka S., Cohen R.J. \& Gray M.D., 2005, MNRAS, 360, 1162

Etoka S., Zijlstra A., Richards A.M., Matsuura M. \& Lagadec E., 2009, ASPC, 404, 311

Etoka S., Gérard E., Richards A. et al., 2010a, evn conf, 6

Etoka S. \& Diamond P.J., 2010b, MNRAS 406, 2218

Etoka S., Gray M.D. \& Fuller G.A., 2012, MNRAS, 423, 647

Fix J.D. \& Mutel R.L, 1985, AJ, 90, 736

Frail D., et al.1996, AJ, 111, 1651 
Goedhart S., Gaylard M.J. \& van der Walt D.J., 2004, MNRAS, 355, 553

Goldsmith P.F., Pandian J,D. \& Deshpande A. A., 2008, ApJ, 680,1132

Gordon K., et al., 2011, AJ, 142, 102

Green J., et al. 2008, MNRAS, 385, 948

Green J.A., McClure-Griffiths N.M., Caswell J.L., Robishaw T.\& Harvey-Smith L., 2012, MNRAS, 425, 2530

Green J., et al., 2015, "Maser Astrometry with VLBI and the SKA", in proceedings of "Advancing Astrophysics with the Square Kilometre Array", PoS(AASKA14)119

Groenewegen M., 2006, A\&A, 448, 181

Gruendl R.A. \& Chu Y.-H.,2009, ApJS, 184, 172

Habing H.J., Sevenster M.N., Messineo M., van de Ven G. \& Kuijken K., 2006, A\&A, 458, 151

Hutawarakorn B., Cohen R.J. \& Brebner G.C., 2002, MNRAS, 330, 349

Imai H., Deguchi S., Nakashima J.-I., Kwok S., \& Diamond P.J., 2013, ApJ, 773, 182

Jackson T., Ivezic Z. \& Knapp G.R., 2002, MNRAS, 337, 749

Lagadec E., et al. 2008, MNRAS, 383, 399

Lagadec E., et al. 2009, MNRAS 396, 598

Lewis B.M., 1992, ApJ, 396, 251

Libert Y., Gérard E. \& Le Bertre T., 2007, MNRAS, 380, 1161

McBride J., Heiles C. \& Elitzur M., 2013, ApJ, 774, 35

Marshall J.R., van Loon J.T., Matsuura M. et al., 2004, MNRAS, 355, 1348

Meixner M., et al., 2006, AJ, 132, 2268

Riebel D., Srinivasan S., Sargent B. \& Meixner M., 2012, ApJ, 753, 71

Robishaw T., Quartaert E. \& Heiles C., 2008, ApJ, 680, 981

Robishaw T., et al., 2015, "Measuring Magnetic Fields Near and Far via the Zeeman Effect", in proceedings of "Advancing Astrophysics with the Square Kilometre Array", PoS(AASKA14)110

Scalise E. Jr. \& Braz M.A., 1982, ApJ, 87, 528

Sevenster M.N. \& Chapman J.M., 2001, ApJ, 546L, 119

Sevenster M.N., van Langevelde H.J., Moody R.A. et al., 2001, A\&A, 366, 481

Sjouwerman L. \& Pihlstrom Y., 2008, ApJ, 681, 1287

Uscanga L., Gómez J.F., Suárez O. \& Miranda L.F., 2012, A\&A, 547, A40

Vlemmings W.H.T. \& van Langevelde H.J., 2007, A\&A, 472, 547

Wright M.M., Gray M.D. \& Diamond P.J., 2004a, MNRAS, 350, 1253

Wright M.M., Gray M.D. \& Diamond P.J., 2004b, MNRAS, 350, 1272

Zijlstra A., et al., 1996, MNRAS, 279, 32 